\title{
Acidic Pharmaceuticals Simultaneous Removal from Water Sample by Magnetic Ionic Liquid Nanoadsorbent 1-Butyl-3-Aminopropyl Imidazolium Tetrachloroferrate Functionalized Graphene Oxide GO-sBapim) $\mathrm{FeCl}_{4}$
}

\author{
Farid Abu Shammala ${ }^{1 *}$ and Barry Chiswell ${ }^{2}$ \\ ${ }^{1}$ Department of Analytical Chemistry, University of Palestine, Palestine \\ ${ }^{2}$ National Research Centre for Environmental Toxicology, University of Queensland, Brisbane, Australia \\ *Corresponding author: Farid Abu Shammala, Faculty of Pharmacy, University of Palestine, El-Zahra City, Palestine. \\ To Cite This Article: Farid Abu Shammala. Acidic Pharmaceuticals Simultaneous Removal from Water Sample by Magnetic Ionic Liquid \\ Nanoadsorbent 1-Butyl-3-Aminopropyl Imidazolium Tetrachloroferrate Functionalized Graphene Oxide GO-sBapim) FeCl. Am J Biomed Sci \& Res. \\ 2019 - 5(1). AJBSR.MS.ID.000875. DOI: 10.34297/AJBSR.2019.05.000875
}

Received: 制August 26, 2019; Published: 制 September 06, 2019

\begin{abstract}
This paper evaluated the removal efficiency of twelve acidic pharmaceuticals by microextraction with 1-butyl-3-aminopropyl imidazolium tetrachloroferrate functionalized graphene oxide GO-(Bapim)FeCl4 nanocomposite. The acidic pharmaceuticals studied were salicylic acid (SYL), clofibric acid (CFA), ibuprofen (IBP), acetaminophen (AAP), gemfibrozil (GFB), fenoprofen (FNP), naproxen (NPX), triclosan (TCS), ketoprofen (KTP), diclofenac (DOF), fenofibrate (FFB), and indomethacin (IDM), which are commonly used as analgesic/anti-inflammatory agents or lipid regulators. The proposed procedure complies with the principles of the green chemistry, since it uses low volumes of easily synthesized MILsbased magnetic extracting phases avoiding the use of toxic solvents. The analytical procedure for the determination of acidic pharmaceuticals in aqueous matrices included solid phase microextraction, liquid chromatographic separation and detection by a triple-quadrupole mass spectrometer. Taking into account the main experimental variables involved, the results obtained are accurate and highly reproducible. The low-cost approach is straightforward, environmentally safe and exhibits high enrichment factors and absolute extraction percentages and satisfactory recoveries.
\end{abstract}

To the best of our knowledge, this is the first time that a MIL-GO is used for analytical purposes in a practical, efficient and environmentally friendly drop-breakup microextraction approach for acidic pharmaceuticals. In the aqueous sample treatment processes, the highest removal rate was observed for ibuprofen and the lowest for acetaminophen, $99 \%+/-1 \%$ and $67 \%+/-2 \%$, respectively. Whereas, the stability of acidic drugs recovery from municipal sewage were the lowest for acetaminophen and salicylic acid.

Keywords: Ionic Liquid; Graphene Oxide; Acidic Pharmaceuticals; Microextraction

\section{Introduction}

Pharmaceutical active compounds have been classified as emerging pollutants of concern because of their detection in aquatic environment and surface waters. Hundreds of tons of pharmaceuticals are annually consumed and dispensed worldwide [1-3]. In urban populated areas, large amount of intact and metabolized drugs enters the aquatic environment via the municipal sewage systems [4-8]. The occurrence of pharmaceuticals in environmental samples was infrequently reported, scientists were beginning to realize that the health of aquatic organisms might be adversely affected by continually exposure to residues of drugs, of which most were biologically active [9-12]. Therefore, it is important to evaluate the fate and concentrations of acidic pharmaceuticals in the water bodies, the related studies on these compounds in drinking water systems are limited, and the efficiencies of advanced processes for their removal need to be evaluated and developed. Acidic pharmaceuticals are an important class of emerging environmental micropollutants, their presence in the aquatic systems such as wastewaters, drinking water and underground water is considered as a major environmental issue [13]

Conventional water treatment processes which have been used for decades for the removal of acidic pharmaceuticals include: sedimentation, coagulation, sand filtration, and chlorination [14]. 
Moreover, advanced water treatment processes have been applied in a water treatment facility for improving the quality of drinking water such as: ozonation, activated carbon and ultra-membrane filtration [15]. Indeed, the most important pathways for the removal of acidic pharmaceuticals during wastewater treatment are biodegradation and abiotic removal by adsorption to the sludge [16-20]. The efficiency of their removal depends on their physico-chemical properties, especially hydrophobicity and biodegradability, and process operating parameters such as $\mathrm{pH}$ and temperature [21]. Several mechanisms including adsorption, dispersion and biological transformations are the main processes driving the attenuation and fate of organic contaminants in groundwater environments during infiltration, these processes are not yet sufficiently understood. Different patterns and kinetics for the removal of the acidic pharmaceuticals can be found; however, there is a general agreement that significant improvement is achieved in the quality of the recovered waters [22-25].

Redox processes in enhanced groundwater enrichment sites are known to affect the elimination of pharmaceutical residues, these processes are known to be temperature-dependent, and catalyzed microbially and temperature variations influence the microbial activity [26]. Under warmer temperatures (greater than $14^{\circ} \mathrm{C}$ ) anoxic conditions prevail, while below $14^{\circ} \mathrm{C}$ anoxic conditions develop. Recent research work investigated the impact of the infiltration water temperature on the redox degradation of carbamazepine, phenazone and phenazone-like pharmaceuticals. The Outcomes suggested that removal during infiltration were shown by many of the pharmaceuticals under aerobic conditions with different degradation rates of removal during infiltration, certain pharmaceuticals compounds such as carbamazepine, were not degraded under aerobic conditions irrespective of its redox state; [27]. These results are in concordance with the results reported by several researchers, such that carbamazepine and another antiepileptic drug, such as primidone, were found to be present in drinking water even infiltration was carried out [27].

Many of the carbon-based nanomaterials, such as: carbon nanotubes, fullerenes, nanofibers, nanohorns, graphene, and their chemically modified analogues, has been investigated as an adsorption material. Due to their properties, these carbon-based nanomaterials have received huge applications in different areas of analytical chemistry and in many other techniques of medicine [32-35] The unique structures of carbon-based nanomaterials allow them to interact with molecules via different non-covalent and covalent forces. These interactions include hydrogen bonding, electrostatic forces, $\pi-\pi$ stacking, van der Waals forces and hydrophobic interactions. Recently, graphene which is a novel and indeed fascinating carbon material, has sparked a tremendous research work from both the theoretical and experimental scientific societies [36]. Due to its extraordinary chemical properties and very high specific sur- face area, carbon-based nanomaterials have been extensively applied as adsorbents in solid phase microextraction techniques and applications.

The ionic liquids have received a wide attention as an alternative to environmentally toxic organic solvents, it is increasingly applied in many techniques in analytical chemistry. These include nanoadsorpant techniques, different methods of chromatography, and electrochemistry science. Ionic liquids have many unique properties that can be changed by the proper choice of the building cations and anions species, these properties including its polarity, hydrophobicity and viscosity. Their chemical nature allows the synthesis of many different ionic liquid solvents with different properties for different applications and areas [37-40]. The impact of ILs in analytical chemistry resulted from their unique properties as negligible vapor pressure associated with high thermal stability, non-molecular solvents, tunable viscosity, good extractability in various inorganic and organic compounds, and miscibility with water and organic solvents. Modified materials, which consist of ionic liquid and magnetic composites, has been applied in many analytical chemistry extraction techniques [41].

One of the problems in the use of ionic liquids in chemistry applications is that at high temperatures the viscosity of the IL is reduced, therefore resulting in a flowing state in which the IL can be lost. Scientists overcomes this problem by the modification of the ILs to polymeric ILs (PILs). The PILs have unique properties such as higher thermal stability compared to monomeric ILs and indeed more resistance to flow. Moreover, PILs are tunable by proper functionalization and modification of the IL monomers, thus changing extractive capabilities along with their physicochemical properties [42-44]. Additionally, the combining the two above-mentioned fields, i.e., carbon-based nanomaterials and MILs, it is possible to design and develop new micro extracting phases with outstanding properties for extraction of twelve acidic pharmaceuticals from water samples. The Functionalized carbon-based nanomaterials with MILs are expected to possess unique adsorbents advantages with tunable microextraction capabilities. This paper provides snapshot of our applications of magnetic ionic liquids (MILs) functionalized graphene oxide GO-MILs in microextraction, as an integral step of sample preparation for the removal of twelve acidic pharmaceuticals from water samples.

The results obtained are accurate and highly reproducible, making it a good alternative approach for routine analysis of were applied to assess and detect the twelve acidic pharmaceuticals in water samples. The low-cost approach is straightforward, environmentally safe and exhibits high enrichment factors and absolute extraction percentages and satisfactory recoveries. To the best of our knowledge, this is the first time that a MIL-GO is used for analytical purposes in a practical, efficient and environmentally friendly for microextraction of acidic pharmaceuticals. 


\section{Materials and Methods}

\section{Reagents and Chemicals}

Analytical standards for the 12 acidic drugs were obtained from Sigma-Aldrich Chemicals. An individual stock solution for each drug at $100.0 \mu \mathrm{g} / \mathrm{mL}$ was prepared in acetonitrile. A mixture of the 12 drugs, each at $10 \mu \mathrm{g} / \mathrm{mL}$, was prepared in acetone for spiking and the preparation of calibration standards. A stock solution and a spiking solution were prepared in acetone.

\section{Sample Preparation}

Each water sample was filtered, under reduced pressure, in the laboratory using a glass fiber filter (Whatman grade GF/C, $1.2 \mu \mathrm{m}$ ) and a layer of Celite, in an all-glass system consisting of a 9-cm sintered base and a 4-L flask. To each 1-L aliquot of the filtrate, 250 $\mu \mathrm{L}$ of the $10 \mu \mathrm{g} / \mathrm{mL}$ acid drug solution and $1 \mathrm{~mL}$ of concentrated $\mathrm{HCl}$ were added and mixed. Meanwhile, a 6-mL, 200-mg MIL-GO nanocomposites cartridge, was conditioned by elution with $5 \mathrm{~mL}$ of methanol, followed by $10 \mathrm{~mL}$ of water at $\mathrm{pH} 2$. A flow rate of 0 to $15 \mathrm{~mL} / \mathrm{min}$ was maintained by adjusting the system vacuum. When the extraction was complete, the cartridges were dried for $1 \mathrm{~min}$ at a vacuum. Each cartridge was washed with $2 \mathrm{~mL}$ of $25 \%$ methanol in water $(\mathrm{v} / \mathrm{v})$ to remove the polar coextractives. After it was further dried under full vacuum for $10 \mathrm{~min}$, the cartridge was eluted with two $2.5-\mathrm{mL}$ aliquots of methanol to remove the adsorbed drugs. The methanol extract was evaporated to ca. $300 \mu \mathrm{L}$ and was back extracted by $5 \mathrm{~mL}$ of methyl tert-butylether (MTBE) in 2-, 1-, 1-, and 1-mL fractions. After passing through a small bed of anhydrous sodium sulfate to remove any residual water, the MILGO nanocomposites extract was evaporated to $50 \mu \mathrm{L}$.

\section{Derivatization}

$100 \mu \mathrm{L}$ of BSTFA with $1 \%$ TMCS was added to the MIL-GO nanocomposites extract and maintained at $60^{\circ} \mathrm{C}$ for $20 \mathrm{~min}$ to form the trimethylsilyl (TMS) derivatives. Then, $3 \mathrm{~mL}$ of water was added, and the products were back extracted into the organic layer with 2 $\mathrm{mL}$ of petroleum ether (b.p. $30-60^{\circ} \mathrm{C}$ ), followed by three $2-\mathrm{mL}$ aliquots of 10:90 dichloromethane in petroleum ether. A centrifuge was used to break up the emulsion. The extract was dried by passing through a pasteur pipette filled with $5 \mathrm{~cm}$ of anhydrous sodium sulfate, evaporated, and adjusted to $1.0 \mathrm{~mL}$ in iso-octane for GC/ MS analysis.

\section{Synthesis of ILs-modified GO composites}

GO was prepared using the well-known modified Hummers method, and MILs was prepared GO through amidation reaction between the carboxyl groups of GO and the amino groups of the MILs. $50 \mathrm{mg}$ of GO was dispersed in $100 \mathrm{~mL}$ of deionized water by ultrasonication for $1 \mathrm{~h}$. After $1 \mathrm{hr} 200 \mathrm{mg}$ of EDC and $160 \mathrm{mg}$ of NHS were added to ensure the homogeneity of the solution. Then, solution was magnetically stirred for $2 \mathrm{~h}$ to activate the carboxyl groups of GO, after that $200 \mathrm{mg}$ of 1-butyl-3-aminopropyl imidazolium tetrachloroferrate was added and the mixture was then ultrasonicated for $60 \mathrm{~min}$, the mixture was stirred at $30^{\circ} \mathrm{C}$ for $1 \mathrm{~h}$. The final product was washed several times with deionized water and methanol.

\section{Instrumentation Measurements}

The morphology characterization of GO and the MIL-GO nanocomposites were obtained on a JEM-2010 transmission electron microscope (TEM, JEOL Ltd., Japan), a HITACHIS-4800 scanning electron microscope (SEM, Hitachi Co. Ltd., Tokyo, Japan). FT-IR spectra analysis were performed on a Thermo Nicolet iS50 Fourier-transform (FT) infrared spectrometer (Madison, WI, USA). Raman spectra analysis were performed on a Nicolet 6700/NXR FT-Raman spectrometer (Thermo Electron, USA) with a laser excitation of $532 \mathrm{~nm}$. The X-ray diffaction (XRD) patterns were recorded with a Bruker D8-Advance diffractometer using $\mathrm{Cu}-\mathrm{K} \alpha(\lambda=0.15418$ $\mathrm{nm}$ ) in the $10-1000(2 \theta)$ range with a step size of 0.020 . Field-emission scanning electron microscopy (FESEM) images were recorded on a Hitachi SU-8010 apparatus. The Brunauer-Emmett-Teller (BET) specific surface area was ascertained by a nitrogen adsorption data measured at 77K on a Quantach-rome NOVA4000 surface area analyzer. X-ray photoelectron spectroscopy (XPS) patterns were recorded on a Thermo Scientific Escalab 250Xi spectrometer with Al-K $\alpha$ (1486.6V). GC/MS analyses of the acidic drugs as their TMS derivatives were performed with a Model 6890 gas chromatograph equipped with a 7683 automatic liquid sampler and a 5973 Mass Selective Detector (Agilent Technologies). Separation of the derivatives was achieved with a $30 \mathrm{~m} \times 0.25 \mathrm{~mm}$ I.D., $0.25-\mu \mathrm{m}$ film thickness, HP-5-MS capillary column using the following temperature program: initial temperature $70^{\circ} \mathrm{C}, 1$-min hold, oven temperature ramps $30^{\circ} \mathrm{C} / \mathrm{min}$ from 70 to $150^{\circ} \mathrm{C}$, and $10^{\circ} \mathrm{C} / \mathrm{min}$ from 150 to $285^{\circ} \mathrm{C}$. The oven temperature was kept at $285^{\circ} \mathrm{C}$ for $6 \mathrm{~min}$. A constant carrier (helium) flow of $0.9 \mathrm{~mL} / \mathrm{min}$ was maintained. The injector temperature was $250^{\circ} \mathrm{C}$. Samples in $1-\mu \mathrm{L}$ aliquots were injected in the splitless mode with a splitless time of $1 \mathrm{~min}$. Electron impact ionization mass spectrometry (EI/MS) was used for the detection of pharmaceutical compounds. Full scan EI mass spectra of the TMS derivatives were recorded over a range from m/z 50 to 450 with electron energy of $70 \mathrm{eV}$. For quantitative analyses, selected ion monitoring (SIM) was used and the characteristic ions chosen for each derivative.

\section{SPE Procedure and Real Sample Preparation}

$20.0 \mathrm{mg}$ of the GO-MILs nanocomposite was packed into a standard filter, to acted as a homemade SPE column. The column was preconditioned with $2 \mathrm{~mL}$ of methanol and $2 \mathrm{~mL}$ of water. $10 \mathrm{~mL}$ of the sample solution was passed through the column at a flow rate of $0.5 \mathrm{~mL} / \mathrm{min}$. Then, the 12 acidic drugs were eluted with $1.0 \mathrm{~mL}$ of methanol and concentrated to dryness under a steam of nitrogen before detection. Fused silica and glass materials were used for the entire procedure to avoid any possible interferences. The 
quantification was based on external calibration with areas relative to the internal standard areas (at least eight calibration standard solutions, $\mathrm{r} 2$ was always above 0.98 ).

\section{Stability Study}

A six samples of $25 \mathrm{~mL}$ from a local sewage was collected in a $50 \mathrm{~mL}$ bottles. Bottle 1 was used as is for immediate blank determination. Bottles 2 to 6 were fortified with a mixture of the 12 acidic drugs to give a nominal concentration of $1.00 \mu \mathrm{g} / \mathrm{L}$ for each drug. Bottle 2 was also extracted immediately after spiking for the determination of drug levels at zero time, while bottles 3 to 6 were kept at $4^{\circ} \mathrm{C}$ in the dark. The drug levels in bottles 3, 4, 5, and 6 were determined 24 h, 48 h, 96 h, and 7 days after spiking, respectively. At each specified time, the entire sample in each bottle was filtered as described above. The filtrate was split into four 1-L samples and each sample was spiked with the surrogate 2,3-D. Due to losses in the filtration process, the fourth sample was slightly less than $1 \mathrm{~L}$ and its volume (between 950 and $980 \mathrm{~mL}$ ) was measured for correction. Each 1-L sample was then acidified, extracted, derivatized, and analyzed as described

before

\section{Results and Discussion}

\section{FT-IR Analysis}

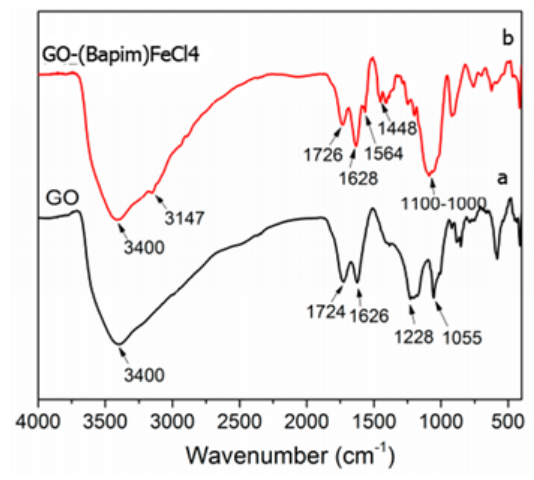

Figure 1: FTIR Spectra of (a) GO, and (b) GO-(Bapim) $\mathrm{FeCl}_{4}$ Nanocomposites.

Figure 1a shows the FTIR spectra of GO and Figure 1b shows the FTIR spectra of GO-(Bapim) $\mathrm{FeCl}_{4}$ nanocomposites. The peak at approximately $3400 \mathrm{~cm}^{-1}$ is caused by stretching vibration of the $-\mathrm{OH}$ group on the surface of GO, the peak at $1726 \mathrm{~cm}^{-1}$ which are caused by the $\mathrm{C}-\mathrm{O}$ stretching vibration of the carboxyl group, the peak at $1626 \mathrm{~cm}^{-1}$ is attributed to the $\mathrm{C}-\mathrm{C}$ bonds in the GO and that at $1628 \mathrm{~cm}^{-1}$ is belongs to the $\mathrm{C}=\mathrm{C}$ bonds in the aromatic groups of Bapim. In Figure $1 \mathrm{~b}$, the peak at $1100 \mathrm{~cm}^{-1}$ is attributed to ring inplane asymmetric stretching of the imidazolium ring. The obvious peak at $1564 \mathrm{~cm}^{-1}$ and the peak at $1448 \mathrm{~cm}^{-1}$ associated with -CONH group. These results suggest that the MILs have been successfully grafted onto the GO surface to form GO-(Bapim) $\mathrm{FeCl}_{4}$ nanocomposites. Thus, FTIR results proved that the functional amine of ionic liquids was chemically bond with carboxyl group on the surface of graphene oxide. Moreover, the imidazolium ring of ionic liquids with alkyl chains offer large $\pi-\pi$ and hydrogen-bond interactions and enhance electrostatic inter-sheet repulsion which result in an increase to the interaction capacity and connectivity between GO and (Bapim) $\mathrm{FeCl}_{4}$.

\section{Raman Spectrum of GO and GO-(Bapim) FeCl4 Nanocom- posite}

Figure 2a shows the Raman spectra of GO and Figure $2 \mathrm{~b}$ shows Raman spectra of GO-(Bapim) $\mathrm{FeCl}_{4}$ nanocomposites, with a laser excitation of $532 \mathrm{~nm}$ was used. In the two samples clearly visible and strong peaks were noticed at approximately $1595 \mathrm{~cm}^{-1}$ and $1353 \mathrm{~cm}^{-1}$ which assigned to the $\mathrm{G}$ and D bands, respectively. We noticed red-shift in the D-band of the Raman spectra of GO-(Bapim) $\mathrm{FeCl}_{4}$ nanocomposites, as shown in Figure 2b. The red-shift phenomena are caused by the bonding between the $\mathrm{C}$ and $\mathrm{N}$ atoms, therefore changes the electronic structure of GO. Indeed, the intensity ratios of the two peaks (ID/IG) demonstrate the extent of defects on the GO surface caused by reaction with (Bapim) $\mathrm{FeCl}_{4}$, and can be used to reflect the extent of covalent binding. The ID/IG ratio of GO and the GO-(Bapim) $\mathrm{FeCl}_{4}$ nanocomposites are 5.0 and 7.0, respectively, corresponding to a slightly increased ratios of ID/IG. And thus an increase in disorder, and indicate the successful formation of amidation reaction between the $\mathrm{GO}$ and (Bapim) $\mathrm{FeCl}_{4}$ to form the final product $\mathrm{GO}$-(Bapim) $\mathrm{FeCl}_{4}$ nanocomposites.

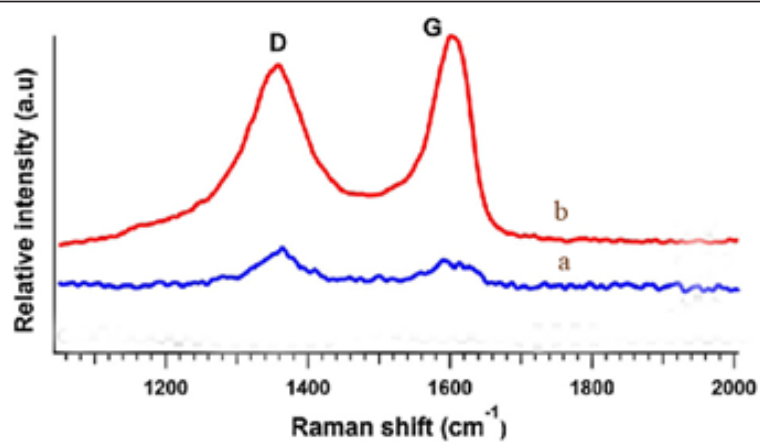

Figure 2: Raman Spectra Spectra of (a) GO, and (b) GO-(Bapim) $\mathrm{FeCl}_{4}$ Nanocomposites.

AFM Image of G0, and GO-(Bapim) FeCl4 Nanocomposites
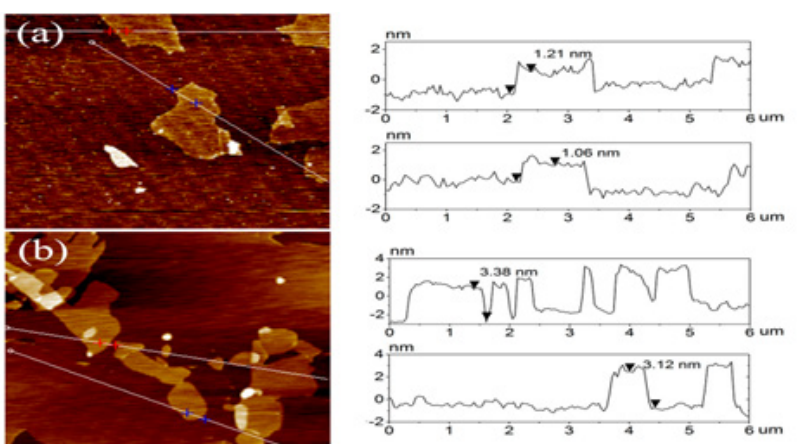

Figure 3: AFM Image of (a) GO, and (b) GO-(Bapim) $\mathrm{FeCl}_{4}$ Nanocomposites. 
Atomic force microscopy images (AFM) image was performed in order to examine the morphology of GO and GO-(Bapim) $\mathrm{FeCl}_{4}$ nanocomposites. AFM images before and after deposition of (Bapim) $\mathrm{FeCl}_{4}$ on the surface of $\mathrm{GO}$ is shown in Figure 3. It can be seen from (Figure 3) a single layer of GO and a single sheet of GO(Bapim) $\mathrm{FeCl}_{4}$ of varying size. (Bapim) $\mathrm{FeCl}_{4}$ are deposited on the surface of GO substrate with clear overlaps. Before deposition of (Bapim) $\mathrm{FeCl}_{4} \mathrm{GO}$ the thickness of GO vary in size from 1.0 to $1.3 \mathrm{~nm}$. However, after functionalization with magnetic ionic liquid (MILs), the thickness of GO single layer sheets are obviously increased to nearly $2.1-2.5 \mathrm{~nm}$, indicating the presence of magnetic ionic liquid (Bapim) $\mathrm{FeCl}_{4}$ on the surface of GO sheet.

\section{X-ray Diffraction (XRD) Analysis}

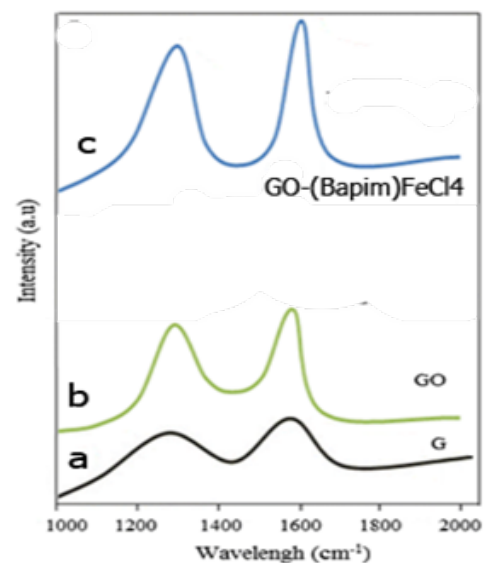

Figure 4: X-ray Diffraction (XRD) Analysis of (a) G (b) GO and (c) $\mathrm{GO}-($ Bapim $) \mathrm{FeCl}_{4}$
X-ray diffraction (XRD) analysis was applied to characterize the crystallographic structure of the fresh and used GO-(Bapim) $\mathrm{FeCl}_{4}$ nanocomposites. As presented in Figure 4, the XRD pattern demonstrating the successful preparation. The used GO-(Bapim) $\mathrm{FeCl}_{4}$ nanocomposites displays the same diffraction pattern as the fresh sample with little decrease in intensity, which indicates that the crystal structure of the $\mathrm{GO}$-(Bapim) $\mathrm{FeCl}_{4}$ nanocomposites is maintained after microeextraction. Moreover, the discernible diffraction peaks at about 9.30 belonging to GO can be detected in the pattern of GO-(Bapim) $\mathrm{FeCl}_{4}$ nanocomposites. This X-ray diffraction (XRD) analysis result indicates the incorporation of (Bapim) $\mathrm{FeCl}_{4}$ into the GO structure.

\section{XPS Scanning Spectrum}

$\mathrm{GO}$ and the GO-(Bapim) $\mathrm{FeCl}_{4}$ nanocomposites were investigated by X-ray photoelectron spectroscopy (XPS). Figure 5 a shows that only the C1s and 01s peaks exist in GO (Figure 5a bottom). The C1s peaks are obtained by coupling the five peaks at 284.8, 285.5 286.8, 287.8, and $288.7 \mathrm{eV}$, assigned for $\mathrm{C}-\mathrm{C}, \mathrm{C}-\mathrm{OH}, \mathrm{C}-\mathrm{O}, \mathrm{C}=\mathrm{O}$, and $\mathrm{O}=\mathrm{C}-\mathrm{OH}$, respectively. The N1s peak can be shown in GO-(Bapim) $\mathrm{FeCl}_{4}$ (Figure $4 \mathrm{a}$ above). Relative to GO, the peak fitting of $\mathrm{C} 1 \mathrm{~s}$ in GO-(Bapim) $\mathrm{FeCl}_{4}$ yields a new functional group (i.e., C-N, Figure $5 \mathrm{c}$ ), which is indicted a successful amidation reaction between (Bapim) $\mathrm{FeCl}_{4}$ and GO. As shown in Figure 5, the N content increases from $0.68 \%$ to $5.10 \%$, while the 0 content decreases, demonstating a covalent amidation bonding between $\mathrm{GO}$ and the (Bapim) $\mathrm{FeCl}_{4}$. Similar results were obtained with the used GO-(Bapim) $\mathrm{FeCl}_{4}$ sample after microextraction of the acidic pharmaceuticals.

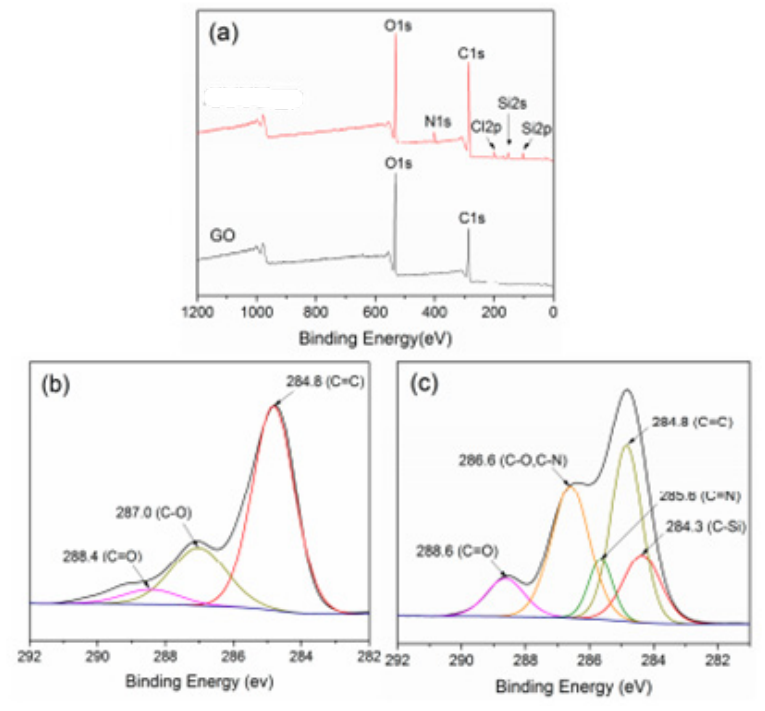

Figure 5: XPS Spectra of GO and GO-(Bapim)FeCl4 Nanocomposites (a) Survey Spectra; (b) C 1s of GO; (c) C 1s of GO-(Bapim)FeCl Nanocomposites.

\section{SEM images and TEM images}

Figure 6a \& 6b shows TEM images of (a) GO, (b) GO-(Bapim) $\mathrm{FeCl}_{4}$ nanocomposites, respectively; and Figure 6c \& 6d depict SEM images of (c) GO and (d) GO-(Bapim) $\mathrm{FeCl}_{4}$ nanocomposites.
As revealed in TEM and SEM images GO possesses a wrinkled single-layer structure with semitransparent flake-like shape. After being grafted by the MILs, the GO-(Bapim) $\mathrm{FeCl}_{4}$ nanocomposites still maintain the lamellar structure shown in Figure 6b and c, which 
ensures that they retain a high specific surface area and high adsorptive performance. An obvious change in the SEM images can be observed after reaction of GO with (Bapim) $\mathrm{FeCl}_{4}$, the large GO sheets are reduced to small pieces, thus giving the appearance of holes with different sizes. This appearance phenomenon can explain the novel nancomposites structure of GO-(Bapim) $\mathrm{FeCl}_{4}$, that have the advantage of the high adsorptive capacity of acidic pharmaceuticals disrupting chemicals.
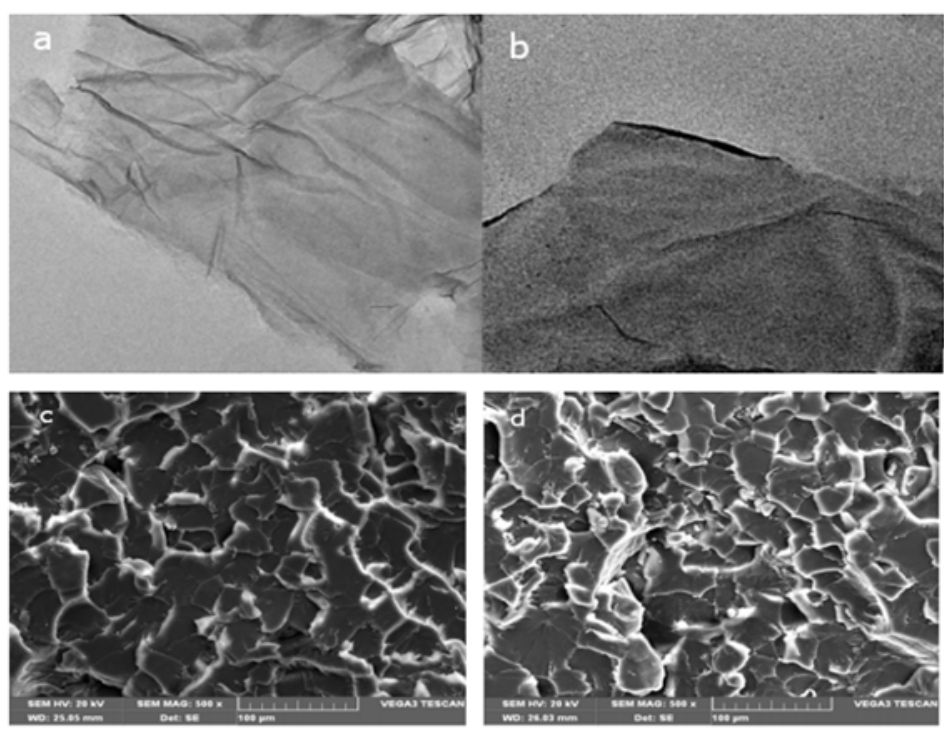

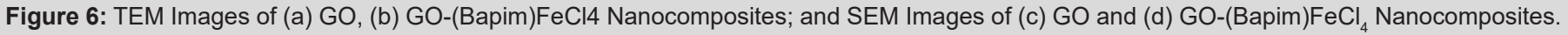

The Removal of Acidic Pharmaceuticals by GO(Bapim) $\mathrm{FeCl}_{4}$ Nanocomposite

(Figure 7) shows GC-MS chromatograms of 12 acidic pharmaceutical extracts of a sample from GO-(Bapim) $\mathrm{FeCl}_{4}$ nanocomposites after recovery. The acidic drug, soluble in polar solvents and remains in protonated (neutral) form at acidic $\mathrm{pH}$. IBP has high melting and boiling points to be directly analyzed by gas chromatography. However, its sublimation increases as temperature increases [40] thus, the vapors of sublimed IBP in injector $\left(\approx 300^{\circ} \mathrm{C}\right)$ are taken to capillary column and carried to mass detector via carrier gas.
(Figure 7) shows the full scan mass spectrum and fragmentation of IBP using electron impact (EI) ionization. For identification of IBP in unknown samples, spectral match with NIST05 library was used whereas optimization and quantification of IBP was carried in selected ion monitoring mode (SIM). Multiple ions were used to enhance the signal thereby the sensitivity of assay. When GC/MS is used for analysis, derivatization of protonic groups in acidic drugs is usually performed in order to improve the chromatography. In previous works, methyl derivatives have been used for the detection of many acidic drugs [40]. Trimethylsilyl (TMS) derivatives have also been used for diclofenac and clofibric acid [41].
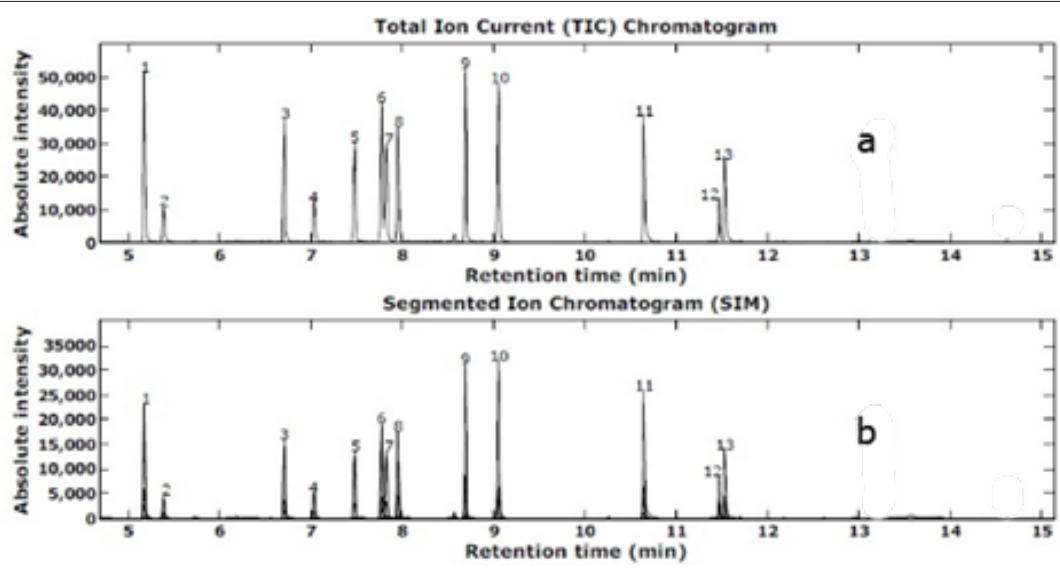

Figure 7: Total GS-MS ion chromatogram for TMS derivatives of 12 standard acidic pharmaceutical; (1) SYL, (2) CFA, (3) IBP, (4) AAP, (5) 2,3-D (internal standard), (6) GFB, (7) FNP, (8) NPX, (9) TCS, (10) KTP, (11) DOF, (12) FFB, and (13) IDM. (b) Total GC-MS lon chromatogram for TMS Derivatives 12 acidic Pharmaceutical Extracts After Recovery from GO-(Bapim)FeCl4 Nanocomposites.

In our work, methyl ester derivatives were not selected, as the procedure would involve a reagent (diazomethane) that is carcinogenic and potentially explosive. As we had been unable to obtain a PFB derivative for acetaminophen, we have also abandoned our investigations into such derivatives for the detection of drugs. BSTFA is a very versatile silylating reagent that converts compounds having active hydrogens such as carboxylic $(-\mathrm{COOH})$ and phenolic $(-\mathrm{OH})$ groups to the corresponding TMS derivatives. Fenofibrate, 
which has no protonic groups, did not react with BSTFA. The other 11 compounds in this study all have at least one of these groups (Table 1). In our case, the formation of a TMS derivative for each drug was confirmed by the observation of its molecular ion under EI/GC/MS conditions. In the case of salicylic acid, the carboxylic and the phenolic groups were both derivatized. However, the $\mathrm{NH}$ group in acetaminophen and diclofenac did not react with BSTFA under the conditions used. Ternes et al. [22] have used a two-step derivatization (i.e., trimethylsilylation of the $\mathrm{OH}$ group followed by trifluoroacylation of the $\mathrm{NH}$ group) to prepare stable derivatives of some neutral drugs for GC/MS analysis.

The second derivatization was not necessary in our work. Even with a free NH group, the TMS derivatives of acetaminophen and diclofenac were stable under GC conditions. TMS derivatives of all the acidic drugs were also stable at $-20^{\circ} \mathrm{C}$ for at least three months.
Characteristic ions of the derivatives, used for the confirmation and quantification of the drugs, are listed in Table 1. As shown in Figure 1, TMS derivatives of the acidic drugs and 2,3-D (surrogate standard) were nicely separated by a HP-5-MS column in a 20-min run. As the peaks were well spaced in the chromatogram, it was therefore easy to divide them into many SIM windows. This allowed the monitoring of one target compound in each window so that detector sensitivity could be optimized. At the same time, several characteristic ions of each compound were monitored to minimize misidentification in very contaminated extracts. However, in order to maintain good sensitivity and chromatography, the inlet must be clean and the column in good condition. Otherwise, tailing peaks, reduced sensitivity, or even a complete disappearance of peaks could occur owing to adsorption of drug derivatives onto active sites in a contaminated GC system

Table 1: The Compared Adsorption Capacity of Standard Acidic Pharmaceuticals from Aqueous Sample with GO-Mils Nanocomposites at Different $\mathrm{Ph}$ Values. Acidic Pharmaceuticals Concentration was $20 \mathrm{Ng} / \mathrm{Ml}$, and Incubation Time, $10 \mathrm{Min}$ Ph Recovery \% of Acidic Pharmaceuticals Using GO-(Bapim)Fecl4 Compositea

\begin{tabular}{|c|c|c|c|c|c|c|c|c|c|c|c|c|}
\hline \multirow{2}{*}{ pH } & \multicolumn{12}{|c|}{ Recovery \% of Acidic Pharmaceuticals using GO-(Bapim)FeCl4 Composite ${ }^{a}$} \\
\hline & SYL & CFA & IBP & AAP & GFB & FNP & NPX & TCS & KTP & DOF & FFB & IDM \\
\hline 3 & 58 & 57 & 56 & 67 & 57 & 59 & 57 & 57 & 64 & 55 & 59 & 54 \\
\hline 4 & 56 & 54 & 57 & 54 & 59 & 57 & 59 & 58 & 66 & 58 & 60 & 58 \\
\hline 5 & 62 & 67 & 67 & 60 & 67 & 67 & 67 & 66 & 65 & 65 & 63 & 58 \\
\hline 6 & 61 & 67 & 77 & 60 & 67 & 67 & 67 & 67 & 65 & 70 & 65 & 66 \\
\hline 7 & 72 & 73 & 86 & 61 & 72 & 77 & 73 & 77 & 74 & 75 & 70 & 71 \\
\hline 8 & 82 & 80 & 94 & 65 & 81 & 82 & 83 & 87 & 85 & 88 & 79 & 78 \\
\hline 9 & 95 & 80 & 95 & 66 & 86 & 88 & 89 & 85 & 94 & 93 & 82 & 98 \\
\hline 10 & 96 & 80 & 99 & 67 & 87 & 86 & 83 & 89 & 95 & 99 & 85 & 99 \\
\hline 11 & 95 & 86 & 99 & 67 & 87 & 87 & 85 & 87 & 98 & 99 & 86 & 92 \\
\hline
\end{tabular}

a: Mean of three determinations.

As shown in Table 1, we find that the GO-(Bapim) $\mathrm{FeCl}_{4}$ nanocomposites possess a superior nanoextraction capacity, mainly for IBP, SYL, TCS, KTP, DOF, and IDM, with the removal efficiencies were above $95 \%$ even to $99 \%$. The results indicate a decreased in carbon chain length of MILs benefits the extraction, and the primary influence on the adsorptive performance of the GO-MIL nanocompos- ites was the nature of the anion, the hydrophobic effect restrained the extraction process in the aqueous system. The results indicated that GO-MIL adsorption capacities especially for CFA, AAP, GFB, FNP, NPX, and TCS are significantly weaker, with the removal efficiencies were between $67 \%$ to $89 \%$ (Figure 7 \& Tables 1-3).

\begin{tabular}{|c|c|c|c|c|c|c|c|c|c|c|c|c|}
\hline \multirow{2}{*}{$\begin{array}{c}\text { Elution } \\
\text { time } \\
\text { (min) }\end{array}$} & \multicolumn{12}{|c|}{ Recovery \% of Acidic Pharmaceuticals using Sing GO-(Bapim)FeCl4 } \\
\hline & SYL & CFA & IBP & AAP & GFB & FNP & NPX & TCS & KTP & DOF & FFB & IDM \\
\hline 1 & 61 & 67 & 87 & 67 & 67 & 67 & 67 & 67 & 57 & 59 & 57 & 57 \\
\hline 2 & 72 & 77 & 91 & 65 & 75 & 74 & 72 & 73 & 76 & 67 & 69 & 63 \\
\hline 3 & 82 & 80 & 94 & 65 & 87 & 87 & 83 & 87 & 85 & 82 & 84 & 83 \\
\hline 4 & 96 & 80 & 94 & 62 & 97 & 96 & 93 & 89 & 85 & 99 & 85 & 99 \\
\hline 5 & 94 & 78 & 92 & 51 & 93 & 94 & 92 & 85 & 88 & 93 & 82 & 98 \\
\hline
\end{tabular}

a: Mean of three determinations.

The influence parameters for $\mathrm{pH}$ values, elution time, and salt concentration were evaluated to find the optimal extraction performance of the GO-(Bapim) $\mathrm{FeCl}_{4}$ for the extraction of acidic pharmaceuticals. In our laboratory, the extraction capability of GO-(Bapim)
$\mathrm{FeCl}_{4}$ for the phenolic EDCs were tested in the range of 3.0-11. As shown in Table 1 , the adsorption capacity $(\mathrm{mg} / \mathrm{g}$ ) clearly increased as the $\mathrm{pH}$ increased from 3.0 to 9.0. Therefore, a pH of 9.0 was chosen for the next adsorption tests. It is noteworthy, that HPLC peak 
areas decreased as the pH became greater than 9.0. Moreover, the chromatogram revealed that acidic pharmaceuticals are relatively independent of changes to the sample solution $\mathrm{pH}$ in the range of $2.0-10$, because they exist as neutral molecules. It is important to mention that the $\mathrm{pH}$ value primarily influences the charge of the ionic liquids and other functional groups such as hydroxyl, carboxyl

Table 3: The compared adsorption capacity of acidic pharmaceuticals from aqueous sample with GO-MILs nanocomposites at different salt concentration. Acidic pharmaceuticals concentration was $20 \mathrm{ng} / \mathrm{mL}$, and Incubation Time, $10 \mathrm{~min}$.

\begin{tabular}{|c|c|c|c|c|c|c|c|c|c|c|c|c|}
\hline \multirow{2}{*}{$\begin{array}{c}\text { Salt } \\
\text { Conc. \% } \\
\text { (w/v) }\end{array}$} & \multicolumn{12}{|c|}{ Recovery \% of Acidic Pharmaceuticals using Sing GO-(Bapim)FeCl4 ${ }^{a}$} \\
\hline & SYL & CFA & IBP & AAP & GFB & FNP & NPX & TCS & KTP & DOF & FFB & IDM \\
\hline $1 \%$ & 94 & 88 & 99 & 67 & 98 & 94 & 98 & 85 & 88 & 96 & 82 & 99 \\
\hline $2 \%$ & 93 & 87 & 94 & 65 & 94 & 85 & 97 & 83 & 80 & 90 & 79 & 93 \\
\hline $3 \%$ & 82 & 76 & 89 & 65 & 80 & 81 & 80 & 80 & 78 & 75 & 68 & 66 \\
\hline $4 \%$ & 67 & 64 & 86 & 65 & 62 & 63 & 67 & 64 & 73 & 68 & 63 & 60 \\
\hline $5 \%$ & 67 & 58 & 77 & 66 & 67 & 67 & 59 & 58 & 68 & 54 & 57 & 54 \\
\hline
\end{tabular}

a: Mean of three determinations

As indicated in Table 3, the salt concentration is a significant factor that influences the extraction capabilities, with $1 \%(\mathrm{w} / \mathrm{v})$ sodium chloride $(\mathrm{NaCl})$ added to the sample solution, the extraction performance of most of the acidic pharmaceuticals reached a maximum. This result may be occurred due to the salt-out effect, which usually promotes extraction. However, when the $\mathrm{NaCl}$ concentration exceeds $2 \%(\mathrm{w} / \mathrm{v})$, the mass transfer process in the solid/liquid interface becomes inhibited from the increased viscosity, leading to a reduction of the diffusion rate of the acidic pharmaceuticals, which decreases the extraction efficiency. For this reason, 1\% (w/v) $\mathrm{NaCl}$ was added to the acidic pharmaceuticals sample solution.

\section{Validation of the Method}

The analytical parameters for the GO-(Bapim) $\mathrm{FeCl}_{4}$ nanocomposites nanoextraction of the acidic pharmaceuticals, such as linearity, correlation coefficients ( $r 2$ ), limits of detection (LODs), limits of quantitation (LOQs) and repeatability were tested under the optimal experimental conditions by using a series of spiked natural water samples. As indicated in Table 4 , the linearity of the acidic pharmaceuticals ranged from $2 \mathrm{ng} \mathrm{mL} \mathrm{m}^{-1}$ to $200 \mathrm{ng} \mathrm{mL}^{-1}$, with the correlation coefficients exceeding 0.99. The LODs and LOQs were defined as the corresponding concentration equivalent to three and ten times the signal-to-noise ratios, in our results ranged from $0.02 \mathrm{ng} \mathrm{mL}^{-1}$ to $0.88 \mathrm{ng} \mathrm{mL}^{-1}$ and from $0.06 \mathrm{ng} \mathrm{mL}^{-1}$ to $2.94 \mathrm{ng} \mathrm{mL}^{-}$ ${ }^{1}$, respectively. The sensitivity of the method, with the use of a UV detector, is quite satisfying, mainly because the detector is easily available to most analytical laboratories. The reproducibility of the method was determined by intra-day RSDs $(n=3)$ and inter-day groups and epoxide on the surface of GO. Table 2 shows the effect of elution time on the extraction performance by changing the washing time from $1 \mathrm{~min}$ to $5 \mathrm{~min}$. our experimental results revealed that the best elution time was 4 . Thus, elution time was set to $4 \mathrm{~min}$ to ensure balance between optimum time and efficiency.
RSDs $(n=3)$ at a spiked sample concentration of $50 \mathrm{ng} \mathrm{mL}^{-1}$. The two RSD values were always less than $8.0 \%$. Indeed, all of our results indicated a high sensitivity and good reproducibility of this new method.

The acidic pharmaceuticals were extracted from sewage GO(Bapim) $\mathrm{FeCl}_{4}$ cartridge, were quantitatively eluted by methanol, certain polar co-extractives such as carboxylic acids and pigments could be removed by a pre-elution step using $2 \mathrm{~mL}$ of $25 \%$ methanol in water. A total ion chromatogram for the TMS derivatives of the 12 acidic drugs and the surrogate 2,3-D. content (e.g. a 1:1 mixture of methanol and water) in the pre-elution step should be avoided, otherwise, losses of many analytes would result. In distilled water samples fortified to 1 and $0.1 \mu \mathrm{g} / \mathrm{L}$, all 12 drugs and the surrogate standard were better than $80 \%$ recovered except for acetaminophen which was only $75 \%$ recovered at $0.1 \mu \mathrm{g} / \mathrm{L}$ (Table $2)$. The standard deviation of replicate $(n=3)$ analyses was found to be small (between $2 \%$ and 5\%). In fortified final effluent samples, similar recoveries were obtained for all compounds but acetaminophen, which could not be recovered at all. The latter observation was again confirmed in the following stability study of the drugs. The response of the Mass Selective Detector, in selected ion monitoring mode, was linear over the concentration range from 25 to $5000 \mathrm{pg} / \mu \mathrm{L}$ for the TMS derivatives of the drugs. Based on a concentration factor of 1000 , the method detection limit for the drugs was estimated at $0.01 \mu \mathrm{g} / \mathrm{L}$ (based on a signal to noise ratio of 5:1) except for indomethacin (MDL $0.02 \mu \mathrm{g} / \mathrm{L}$ ). This method is not applicable to the determination of acetaminophen in sewage samples (Table 4).

\begin{tabular}{|c|c|c|c|c|c|c|c|}
\hline Analytes & $\begin{array}{c}\text { Linear Range } \\
\text { (ng/mL) }\end{array}$ & Calibration Equations & r2 & LODs (ng/mL) & LOQ (ng/mL) & Intra-day RSDs (\%) & Inter-day RSDs (\%) \\
\hline SYL & $10-200$ & $y=25 \pm 1 x-57$ & 0.9944 & 0.88 & 2.94 & 8 & 8.1 \\
\hline CFA & $2-100$ & $y=752 \pm 16 x+1012$ & 0.9976 & 0.1 & 0.09 & 3.8 & 5.6 \\
\hline IBP & $2-200$ & $y=436 \pm 6 x-27$ & 0.9989 & 0.08 & 0.16 & 3.9 & 6.2 \\
\hline AAP & $5-200$ & $y=246 \pm 7 x-1186$ & 0.9967 & 0.03 & 0.32 & 6.4 & 7.3 \\
\hline
\end{tabular}




\begin{tabular}{|c|c|c|c|c|c|c|c|}
\hline GFB & $5-200$ & $y=264 \pm 7 x-48$ & 0.9971 & 0.08 & 0.28 & 5 & 7.1 \\
\hline FNP & $5-200$ & $y=182 \pm 6 x+230$ & 0.9957 & 0.12 & 0.39 & 6.2 & 6.6 \\
\hline NPX & $10-200$ & $y=46 \pm 2 x+2070$ & 0.9948 & 0.24 & 0.8 & 6.6 & 7.2 \\
\hline TCS & $10-200$ & $y=48 \pm 2 x-26$ & 0.9939 & 0.44 & 1.42 & 4.6 & 5.8 \\
\hline KTP & $5-200$ & $y=182 \pm 6 x+230$ & 0.9957 & 0.12 & 0.39 & 6.2 & 6.6 \\
\hline DOF & $10-200$ & $y=168 \pm 2 x-28$ & 0.9932 & 0.45 & 1.4 & 4.6 & 5.7 \\
\hline FFB & $5-200$ & $y=128 \pm 2 x-24$ & 0.993 & 0.44 & 1.42 & 5.6 & 6.8 \\
\hline IDM & $10-200$ & $y=48 \pm 2 x-26$ & 0.994 & 0.43 & 1.46 & 4.9 & 5.7 \\
\hline
\end{tabular}

\section{The Stability of Acidic Drugs in Municipal Sewage}

The stability of acidic drugs in a biologically active matrix such as municipal sewage is a crucial factor that could affect the reliability of the method. Table 4, shows the stability of acidic drugs in sewage samples. The initial concentrations of acidic pharmaceutical were higher than the nominal concentration of $5 \mu \mathrm{g} / \mathrm{L}$. Indeed, there are very few reports on the stability of acidic drugs in sewage samples under storage conditions. Using a bulk effluent sample, we have tested the stability of the acidic drugs, kept at $4{ }^{\circ} \mathrm{C}$ in the dark, for a storage period of up to seven days. The initial concentrations were determined immediately after fortification and were used to calculate the \% recoveries of the drugs in the fortified subsamples analyzed at a later time. Due to the presence of several acidic drugs in the blank, the initial concentrations of acidic pharmaceutical were higher than the nominal concentration of $5 \mu \mathrm{g} / \mathrm{L}$. The acetaminophen has very low recovery even at zero time, which suggests that this compound degrades rapidly in sewage samples. Salicylic acid was also found to degrade rapidly in sewage samples; as little as $16 \%$ was recovered after a storage period of $24 \mathrm{~h}$. No salicylic acid could be recovered if the sample had been stored for two days or longer. In contrast, the other 10 drugs were fairly stable under similar conditions. Their recoveries were better than $85 \%$ and $73 \%$ after 1 and 2 days of storage, respectively. Since the stability study was carried out with a single effluent sample, our results might not be entirely representative. Nevertheless, our findings were consistent with the low detection of acetaminophen in all the sewage (Table 5).

Table 5: The stability of acidic drugs in sewage samples. The initial concentrations of acidic pharmaceutical were higher than the nominal concentration of $5 \mu \mathrm{g} / \mathrm{L}$.

\begin{tabular}{|c|c|c|c|c|c|c|c|c|c|c|c|c|}
\hline \multirow{2}{*}{ Time, hr } & \multicolumn{12}{|c|}{ Recovery \% of Acidic Pharmaceuticals using sing GO-(Bapim)FeCl4 ${ }^{a}$} \\
\hline & SYL & CFA & IBP & AAP & GFB & FNP & NPX & TCS & KTP & DOF & FFB & IDM \\
\hline 1 & 51 & 67 & 67 & 17 & 67 & 67 & 67 & 67 & 57 & 59 & 57 & 57 \\
\hline 6 & 42 & 77 & 71 & 15 & 75 & 74 & 72 & 73 & 76 & 67 & 69 & 63 \\
\hline 12 & 22 & 80 & 84 & 14 & 87 & 87 & 83 & 87 & 85 & 82 & 84 & 83 \\
\hline 24 & 16 & 75 & 74 & 11 & 87 & 86 & 73 & 85 & 85 & 79 & 85 & 85 \\
\hline 48 & 15 & 78 & 72 & 5 & 73 & 74 & 72 & 75 & 78 & 73 & 75 & 78 \\
\hline
\end{tabular}

\section{Conclusion}

This research highlights the preparation of novel types of GOMILs nanocomposite for use in SPME of acidic drugs from aqueous solution. In our research, a magnetic ionic liquid modified graphene oxide nanocomposite was prepared through a direct amidation reaction between GO and MIL. The prepared nanocomposites were used as the nanoadsorbent in a fixed-bed column, which possessed the advantages of low column pressure and high nanoadsorption capacity. Moreover, the system was successfully applied to the microextraction of acidic pharmaceuticals from aqueous samples with good reproducibility, wide linear range and low LODs using a standard GC-MS technique. The proposed method provides a reliable method for the removal and determination of acidic pharmaceuticals in aqueous solution, which can be applied in water treatment and the regulation of supplies. All the new findings of the laboratory studies described in this paper help to better understand of the recovery and fat of acidic pharmaceuticals in municipal sewage water.

\section{References}

1. Androezzi R, Raffaele M, Nicklas P (2003) Pharmaceuticals in STP effluents and their solar photodegradation in aquatic environment. Chemosphere 50(10): 1319-1330.

2. Buser HR, Müller MD, Theobald N (1998) Occurrence of the pharmaceutical drug clofibric acid and the herbicide mecoprop in various Swiss lakes and the North Sea. Environ Sci Technol 32(1): 188-192.

3. Buser HR, Poiger T, Müller MD (1998) Occurrence and fate of the drug diclofenac in surface waters: rapid photodegradation in a lake. Environ Sci Technol 32(32): 3449-3456.

4. Buser HR, Poiger T, Müller MD (1999) Occurrence and environmental behavior of the chiral pharmaceutical drug ibuprofen in surface waters and in wastewater. Environ Sci Technol 33(15): 2529-2535.

5. Daughton CG, Ternes TA (1999) Pharmaceuticals and personal care products in the environment: agents of subtle change? Environ Health Perspect. 107(1 6): 907-938.

6. Halling Sorensen B, Nors Nielsen S, Lanzky PF, Ingerslev F, Holten Lutzhoft HC, et al. (1998) Occurrence, fate and effects of pharmaceuticals substances in the environment-a review. Chemosphere 36(2): 357-393.

7. Heberer Th (2002) Occurrence, fate, and removal of pharmaceutical residues in the aquatic environment: a review of recent research data. Toxicol Lett 131(1-2): 5-17. 
8. Jones OAH, Voulvoulis N, Lester JN (2001) Human pharmaceuticals in the aquatic environment a review. Environ Technol 22(12): 1383-1394.

9. Jones OAH, Voulvoulis N, Lester JN (2002) Aquatic environmental assessment of the top 25 English prescription pharmaceuticals. Water Res 36(20): 5013-5022.

10. Kolpin DW, Furlong ET, Meyer MT, Thurman EM, Zaugg SD et al. (2002) Pharmaceuticals, hormones, and other organic wastewater contaminants in U.S. streams, 1999-2000: a national reconnaissance. Environ Sci Technol 36(6): 1202-1211.

11. Koutsouba V, Herberer Th, Fuhrmann B, Schmidt-Baumler K, Tsipi D et al. (2003) Determination of polar pharmaceuticals in sewage water of Greece by gas chromatography-mass spectrometry. Chemosphere 51(2): 69-75.

12. Kümmerer K (2001) Drugs in the environment: emission of drugs, diagnostic aids and disinfectants into wastewater by hospitals in relation to other sources a review. Chemosphere 45(6-7): 957-969.

13. Lindqvist N, Tunkanen T, Kronberg L (2005) Occurrence of acidic pharmaceuticals in raw and treated sewages and in receiving waters. Water Res 39(11): 2219-2228.

14. Vieno N, Tuhkanen T, Kronberg L (2006) Removal of Pharmaceuticals in Drinking Water Treatment: Effect of Chemical Coagulation. Envirnmental Technology 27(2): 183-192.

15. Abdel Shafy HI, Mansour MSM (2013) Issue of pharmaceutical compounds in water and wastewater. Egyptian J Chem 56(5,6): 449-471.

16. Pronk W, Palmquist H, Biebow M, Boller M (2006) Nanofiltration for the separation of pharmaceuticals from nutrients in source-separated urine. Water Res 40(7): 1405-1412.

17. Metcalfe CD, Koenig BG, Miao XS, Ternes TA, Struger J (2002) Prescription and non-prescription drugs in the Canadian aquatic environment. Presented at Assessment and Management of Pharmaceuticals and Personal Care Products in the Canadian Environment Workshop Niagara on the lake. Ontario February 24-27.

18. Miao XS, Koenig BG, Metcalfe CD (2002) Analysis of acidic drugs in the effluents of sewage treatment plants using liquid chromatography-electrospray ionization tandem mass spectrometry. J Chromatogr A 952(12): 139-147.

19. Öllers S, Singer HP, Fässler P, Müller SR (2001) Simultaneous quantification of neutral and acidic pharmaceuticals and pesticides at the low-ng/l level in surface and waste water. J Chromatogr A 911(2): 225-234.

20. Patterson DB, Brumley WC, Kelliher V, Ferguson PL (2002) Application of US EPA methods to the analysis of pharmaceuticals and personal care products in the environment: determination of clofibric acid in sewage effluent by GC-MS. Amer Lab July pp. 20-28.

21. Sacher F, Lange FT, Brauch HJ, Blankenhorn I (2001) Pharmaceuticals in ground waters. Analytical methods and results of a monitoring program in Baden Württemberg. Germany J Chromatogr A 938(1-2): 199-210.

22. Ternes TA (1998) Occurrence of drugs in German sewage treatment plants and rivers. Water Res 32: 3245-3260.

23. Ternes TA, Hirsch R, Mueller J, Haberer K (1998) Methods for the determination of neutral drugs as well as betablockers and $\mathrm{B2}$ sympathomimetics in aqueous matrices using GC/MS and LC/MS/MS. Fresenius J Anal Chem 362: 329-340.

24. Ternes TA, Meisenheimer M, McDowell D, Sacher F, Brauch HJ et al. (2002) Removal of pharmaceuticals during drinking water treatment. Environ Sci Technol 36(17): 3855-3863.
25. Ternes TA, Stumpf M, Schuppert B, Haberer K (1998) Simultaneous determination of antiseptics and acid drugs in sewage and river water. Vom Wasser 90: 295-309.

26. Prommer H, Stuyfzand P (2005) Identification of Temperature Dependent Water Quality Changes during a Deep Well Injection Experiment in a Pyritic Aquifer. Environmental Science and Technol 39(7): 2200-2209.

27. Massmann G, Duennbier U, Heberer T, Taute T (2008) Behaviour and redox sensitivity of pharmaceutical residues during bank filtration Investigation of residues of phenazone type analgesics. Chemosphere 71(8): 1476-1485.

28. Zhu Y (2010) Graphene and graphene oxide: synthesis, properties, and applications. Adv Mater 22(35): 3906-3924.

29. Stoller MD, Park S, Zhu Y, An J Ruoff R S (2008) Graphene-based ultracapacitors. Nano Lett 8(10): 3498-3502.

30. Dikin DA (2007) Preparation and characterization of graphene oxide paper. Nature 448: 457-460.

31. Liu Z, Robinson JT, Sun X, Dai H (2008) Gylated nanographene oxide for delivery of water-insoluble cancer drugs. J Am Chem Soc 130(33): 10876-10877.

32. Wang J, Chen Z, Chen B (2014) Adsorption of polycyclic aromatic hydrocarbons by graphene and graphene oxide nanosheets. Environ Sci Technol 48(9): 4817-4825.

33. Plechkova NV, Seddon KR (2008) Applications of ionic liquids in the chemical industry. Chem Soc Rev 37: 123-150.

34. Anderson JL, Ding R, Ellern A, Armstrong DW (2005) Structure and properties of high stability geminal dicationic ionic liquids. J Am Chem Soc 127(2): 593-604

35. Fan T (2016) Fabricating of high-performance functional graphene fibers for micro-capacitive energy storage. Sci Rep 6: 29534.

36. Zhou X, Zhu A, Shi G (2015) Selective extraction and analysis of catecholamines in rat blood micro dialysate by polymeric ionic liquid-diphenylboric acid-packed capillary column and fast separation in high-performance liquid chromatography-electrochemical detector. J Chromatogr A 1409: 125-131.

37. Tamilarasan P, Ramaprabhu S (2015) Integration of polymerized ionic liquid with graphene for enhanced $\mathrm{CO} 2$ adsorption. J Mater Chem A 3: 101-108.

38. Ye YS (2011) A new graphene-modified protic ionic liquid-based composite membrane for solid polymer electrolytes. J Mater Chem 21: 10448-10453.

39. Li B, Rooney DW, Zhang N, Sun K (2013) An in situ ionic-liquid-assisted synthetic approach to iron fluoride/graphene hybrid nanostructures as superior cathode materials for lithium ion batteries. ACS Appl Mater Interfaces 5(11): 5057-5063.

40. Valentini F (2012) Oxidized graphene in ionic liquids for assembling chemically modified electrodes: a structural and electrochemical characterization study. Anal Chem 84(13): 5823-5831.

41. Rachel A, Maxwell J (2012) An examination of thermodynamics of fusion vaporization and sublimation of ibuprofen and naproxen by correlation gas chromatography. Journal of pharmaceutical Science 101(2): 805814.

42. Terner TA (2002) Removal of pharmaceuticals during drinking water treatment. Environ Sci Technol 36(17): 3855-3863. 